\title{
Cosmology from String Theory
}

\author{
Luis Anchordoqui, ${ }^{1}$ Haim Goldberg, ${ }^{2}$ Satoshi Nawata, ${ }^{1}$ and Carlos Nuñez ${ }^{3}$ \\ ${ }^{1}$ Department of Physics, \\ University of Wisconsin-Milwaukee, Milwaukee, WI 53201 \\ ${ }^{2}$ Department of Physics, \\ Northeastern University, Boston, MA 02115 \\ 3 Department of Physics, \\ University of Swansea, Singleton Park, Swansea SA2 8PP, UK
}

(Dated: April 2007)

\begin{abstract}
We explore the cosmological content of Salam-Sezgin six dimensional supergravity, and find a solution to the field equations in qualitative agreement with observation of distant supernovae, primordial nucleosynthesis abundances, and recent measurements of the cosmic microwave background. The carrier of the acceleration in the present de Sitter epoch is a quintessence field slowly rolling down its exponential potential. Intrinsic to this model is a second modulus which is automatically stabilized and acts as a source of cold dark matter, with a mass proportional to an exponential function of the quintessence field (hence realizing VAMP models within a String context). However, any attempt to saturate the present cold dark matter component in this manner leads to unacceptable deviations from cosmological data - a numerical study reveals that this source can account for up to about $7 \%$ of the total cold dark matter budget. We also show that (1) the model will support a de Sitter energy in agreement with observation at the expense of a miniscule breaking of supersymmetry in the compact space; (2) variations in the fine structure constant are controlled by the stabilized modulus and are negligible; (3) "fifth" forces are carried by the stabilized modulus and are short range; (4) the long time behavior of the model in four dimensions is that of a Robertson-Walker universe with a constant expansion rate $(w=-1 / 3)$. Finally, we present a String theory background by lifting our six dimensional cosmological solution to ten dimensions.
\end{abstract}




\section{GENERAL IDEA}

The mechanism involved in generating a very small cosmological constant that satisfies 't Hooft naturalness is one of the most pressing questions in contemporary physics. Recent observations of distant Type Ia supernovae [1] strongly indicate that the universe is expanding in an accelerating phase, with an effective de-Sitter (dS) constant $H$ that nearly saturates the upper bound given by the present-day value of the Hubble constant, i.e., $H \lesssim H_{0} \sim 10^{-33} \mathrm{eV}$. According to the Einstein field equations, $H$ provides a measure of the scalar curvature of the space and is related to the vacuum energy density $\rho_{\text {vac }}$ through Friedmann's equation, $3 M_{\mathrm{Pl}}^{2} H^{2} \sim \rho_{\mathrm{vac}}$, where $M_{\mathrm{Pl}} \simeq 2.4 \times 10^{18} \mathrm{GeV}$ is the reduced Planck mass. However, the "natural" value of $\rho_{\mathrm{vac}}$ coming from the zero-point energies of known elementary particles is found to be at least $\rho_{\mathrm{vac}} \sim \mathrm{TeV}^{4}$. Substitution of this value of $\rho_{\mathrm{vac}}$ into Friedmann's equation yields $H \gtrsim 10^{-3} \mathrm{eV}$, grossly inconsistent with the set of supernova (SN) observations. The absence of a mechanism in agreement with 't Hooft naturalness criteria then centers on the following question: why is the vacuum energy needed by the Einstein field equations 120 orders of magnitude smaller than any "natural" cut-off scale in effective field theory of particle interactions, but not zero?

Nowadays, the most popular framework which can address aspects of this question is the anthropic approach, in which the fundamental constants are not determined through fundamental reasons, but rather because such values are necessary for life (and hence intelligent observers to measure the constants) [2]. Of course, in order to implement this idea in a concrete physical theory, it is necessary to postulate a multiverse in which fundamental physical parameters can take different values. Recent investigations in String theory have applied a statistical approach to the enormous "landscape" of metastable vacua present in the theory [3]. A vast ensemble of metastable vacua with a small positive effective cosmological constant that can accommodate the low energy effective field theory of the Standard Model (SM) have been found. Therefore, the idea of a string landscape has been used to proposed a concrete implementation of the anthropic principle.

Nevertheless, the compactification of a String/M-theory background to a four dimensional solution undergoing accelerating expansion has proved to be exceedingly difficult. The obstruction to finding dS solutions in the low energy equations of String/M theory is well known and summarized in the no-go theorem of [4]. This theorem states that in a $D$-dimensional theory of gravity, in which $(a)$ the action is linear in the Ricci scalar curvature $(b)$ the potential for the matter fields is non-positive and $(c)$ the massless fields have positive defined kinetic terms, there are no (dynamical) compactifications of the form: $d s_{D}^{2}=\Omega^{2}(y)\left(d x_{d}^{2}+\hat{g}_{m n} d y^{n} d y^{m}\right)$, if the $d$ dimensional space has Minkowski $S O(1, d-1)$ or $\mathrm{d} S$ $S O(1, d)$ isometries and its $d$ dimensional gravitational constant is finite (i.e., the internal space has finite volume). The conclusions of the theorem can be circumvented if some of its hypotheses are not satisfied. Examples where the hypotheses can be relaxed exist: $(i)$ one can find solutions in which not all of the internal dimensions are compact [5]; $(i i)$ one may try to find a solution breaking Minkowski or de Sitter invariance [6]; (iii) one may try to add negative tension matter (e.g., in the form of orientifold planes) [7]; (iv) one can even appeal to some intrincate String dynamics [8].

Salam-Sezgin six dimensional supergravity model [9] provides a specific example where the no-go theorem is not at work, because when their model is lifted to $M$ theory the internal space is found to be non-compact [10]. The lower dimensional perspective of this, is that in six dimensions the potential can be positive. This model has perhaps attracted 
the most attention because of the wide range of its phenomenological applications [11]. In this article we examine the cosmological implications of such a supergravity model during the epochs subsequent to primordial nucleosynthesis. We derive a solution of Einstein field equations which is in qualitative agreement with luminosity distance measurements of Type Ia supernovae [1], primordial nucleosynthesis abundances [12], data from the Sloan Digital Sky Survey (SDSS) [13], and the most recent measurements from the Wilkinson Microwave Anisotropy Probe (WMAP) satellite [14]. The observed acceleration of the universe is driven by the "dark energy" associated to a scalar field slowly rolling down its exponential potential (i.e., kinetic energy density < potential energy density $\equiv$ negative pressure) [15]. Very interestingly, the resulting cosmological model also predicts a cold dark matter (CDM) candidate. In analogy with the phenomenological proposal of [16], such a nonbaryonic matter interacts with the dark energy field and therefore the mass of the CDM particles evolves with the exponential dark energy potential. However, an attempt to saturate the present CDM component in this manner leads to gross deviations from present cosmological data. We will show that this type of CDM can account for up to about $7 \%$ of the total CDM budget. Generalizations of our scenario (using supergravities with more fields) might account for the rest.

\section{SALAM-SEZGIN COSMOLOGY}

We begin with the action of Salam-Sezgin six dimensional supergravity [9], setting to zero the fermionic terms in the background (of course fermionic excitations will arise from fluctuations),

$$
S=\frac{1}{4 \kappa^{2}} \int d^{6} x \sqrt{g_{6}}\left[R-\kappa^{2}\left(\partial_{M} \sigma\right)^{2}-\kappa^{2} e^{\kappa \sigma} F_{M N}^{2}-\frac{2 g^{2}}{\kappa^{2}} e^{-\kappa \sigma}-\frac{\kappa^{2}}{3} e^{2 \kappa \sigma} G_{M N P}^{2}\right] .
$$

Here, $g_{6}=\operatorname{det} g_{M N}, R$ is the Ricci scalar of $g_{M N}, F_{M N}=\partial_{[M} A_{N]}, G_{M N P}=\partial_{[M} B_{N P]}+$ $\kappa A_{[M} F_{N P]}$, and capital Latin indices run from 0 to 5 . A re-scaling of the constants: $G_{6} \equiv 2 \kappa^{2}$, $\phi \equiv-\kappa \sigma$ and $\xi \equiv 4 g^{2}$ leads to

$$
S=\frac{1}{2 G_{6}} \int d^{6} x \sqrt{g_{6}}\left[R-\left(\partial_{M} \phi\right)^{2}-\frac{\xi}{G_{6}} e^{\phi}-\frac{G_{6}}{2} e^{-\phi} F_{M N}^{2}-\frac{G_{6}}{6} e^{-2 \phi} G_{M N P}^{2}\right] .
$$

The length dimensions of the fields are: $\left[G_{6}\right]=L^{4},[\xi]=L^{2},[\phi]=\left[g_{M N}^{2}\right]=1,\left[A_{M}^{2}\right]=L^{-4}$, and $\left[F_{M N}^{2}\right]=\left[G_{M N P}^{2}\right]=L^{-6}$.

Now, we consider a spontaneous compactification from six dimension to four dimension. To this end, we take the six dimensional manifold $M$ to be a direct product of 4 Minkowski directions (hereafter denoted by $N_{1}$ ) and a compact orientable two dimensional manifold $N_{2}$ with constant curvature. Without loss of generality, we can set $N_{2}$ to be a sphere $S^{2}$, or a $\Sigma_{2}$ hyperbolic manifold with arbitrary genus. The metric on $M$ locally takes the form

$$
d s_{6}^{2}=d s_{4}(t, \vec{x})^{2}+e^{2 f(t, \vec{x})} d \sigma^{2}, \quad d \sigma^{2}= \begin{cases}r_{c}^{2}\left(d \vartheta^{2}+\sin ^{2} \vartheta d \varphi^{2}\right) & \text { for } S^{2} \\ r_{c}^{2}\left(d \vartheta^{2}+\sinh ^{2} \vartheta d \varphi^{2}\right) & \text { for } \Sigma_{2},\end{cases}
$$

where $(t, \vec{x})$ denotes a local coordinate system in $N_{1}, r_{c}$ is the compactification radius of $N_{2}$. We assume that the scalar field $\phi$ is only dependent on the point of $N_{1}$, i.e., $\phi=\phi(t, \vec{x})$. We further assume that the gauge field $A_{M}$ is excited on $N_{2}$ and is of the form

$$
A_{\varphi}= \begin{cases}b \cos \vartheta & \left(S^{2}\right) \\ b \cosh \vartheta & \left(\Sigma_{2}\right)\end{cases}
$$


This is the monopole configuration detailed by Salam-Sezgin [9]. Since we set the KalbRamond field $B_{N P}=0$ and the term $A_{[M} F_{N P]}$ vanishes on $N_{2}, G_{M N P}=0$. The field strength becomes

$$
F_{M N}^{2}=2 b^{2} e^{-4 f} / r_{c}^{4} .
$$

Taking the variation of the gauge field $A_{M}$ in Eq. (2) we obtain the Maxwell equation

$$
\partial_{M}\left[\sqrt{g_{4}} \sqrt{g_{\sigma}} e^{2 f-\phi} F^{M N}\right]=0 .
$$

It is easily seen that the field strengths in Eq. (5) satisfy Eq. (6).

With this in mind, the Ricci scalar reduces to [17]

$$
R[M]=R\left[N_{1}\right]+e^{-2 f} R\left[N_{2}\right]-4 \square f-6\left(\partial_{\mu} f\right)^{2},
$$

where $R[M], R\left[N_{1}\right]$, and $R\left[N_{2}\right]$ denote the Ricci scalars of the manifolds $M, N_{1}$, and $N_{2}$; respectively. (Greek indices run from 0 to 3 ). The Ricci scalar of $N_{2}$ reads

$$
R\left[N_{2}\right]= \begin{cases}+2 / r_{c}^{2} & \left(S^{2}\right) \\ -2 / r_{c}^{2} & \left(\Sigma_{2}\right)\end{cases}
$$

To simplify the notation, from now on, $R_{1}$ and $R_{2}$ indicate $R\left[N_{1}\right]$ and $R\left[N_{2}\right]$, respectively. The determinant of the metric can be written as $\sqrt{g_{6}}=e^{2 f} \sqrt{g_{4}} \sqrt{g_{\sigma}}$, where $g_{4}=\operatorname{det} g_{\mu \nu}$ and $g_{\sigma}$ is the determinant of the metric of $N_{2}$ excluding the factor $e^{2 f}$. We define the gravitational constant in the four dimension as

$$
\frac{1}{G_{4}} \equiv \frac{M_{\mathrm{Pl}}^{2}}{2}=\frac{1}{2 G_{6}} \int d^{2} \sigma \sqrt{g_{\sigma}}=\frac{2 \pi r_{c}^{2}}{G_{6}}
$$

Hence, by using the field configuration given in Eq. (4) we can re-write the action in Eq. (2) as follows

$$
S=\frac{1}{G_{4}} \int d^{4} x \sqrt{g_{4}}\left\{e^{2 f}\left[R_{1}+e^{-2 f} R_{2}+2\left(\partial_{\mu} f\right)^{2}-\left(\partial_{\mu} \phi\right)^{2}\right]-\frac{\xi}{G_{6}} e^{2 f+\phi}-\frac{G_{6} b^{2}}{r_{c}^{4}} e^{-2 f-\phi}\right\} .
$$

Let us consider now a rescaling of the metric of $N_{1}: \hat{g}_{\mu \nu} \equiv e^{2 f} g_{\mu \nu}$ and $\sqrt{\hat{g}_{4}}=e^{4 f} \sqrt{g_{4}}$. Such a transformation brings the theory into the Einstein conformal frame where the action given in Eq. (10) takes the form

$$
S=\frac{1}{G_{4}} \int d^{4} x \sqrt{\hat{g}_{4}}\left[R\left[\hat{g}_{4}\right]-4\left(\partial_{\mu} f\right)^{2}-\left(\partial_{\mu} \phi\right)^{2}-\frac{\xi}{G_{6}} e^{-2 f+\phi}-\frac{G_{6} b^{2}}{r_{c}^{4}} e^{-6 f-\phi}+e^{-4 f} R_{2}\right] .
$$

The four dimensional Lagrangian is then

$$
L=\frac{\sqrt{g}}{G_{4}}\left[R-4\left(\partial_{\mu} f\right)^{2}-\left(\partial_{\mu} \phi\right)^{2}-V(f, \phi)\right]
$$

with

$$
V(f, \phi) \equiv \frac{\xi}{G_{6}} e^{-2 f+\phi}+\frac{G_{6} b^{2}}{r_{c}^{4}} e^{-6 f-\phi}-e^{-4 f} R_{2},
$$

where to simplify the notation we have defined: $g \equiv \hat{g}_{4}$ and $R \equiv R\left[\hat{g}_{4}\right]$. 
Let us now define a new orthogonal basis, $X \equiv(\phi+2 f) / \sqrt{G}_{4}$ and $Y \equiv(\phi-2 f) / \sqrt{G}_{4}$, so that the kinetic energy terms in the Lagrangian are both canonical, i.e.,

$$
L=\sqrt{g}\left[\frac{R}{G_{4}}-\frac{1}{2}(\partial X)^{2}-\frac{1}{2}(\partial Y)^{2}-\tilde{V}(X, Y)\right],
$$

where the potential $\tilde{V}(X, Y) \equiv V(f, \phi) / G_{4}$ can be re-written (after some elementary algebra) as [18]

$$
\tilde{V}(X, Y)=\frac{e^{\sqrt{G_{4}} Y}}{G_{4}}\left[\frac{G_{6} b^{2}}{r_{c}^{4}} e^{-2 \sqrt{G_{4}} X}-R_{2} e^{-\sqrt{G_{4}} X}+\frac{\xi}{G_{6}}\right] .
$$

The field equations are

$$
\begin{aligned}
R_{\mu \nu}-\frac{1}{2} g_{\mu \nu} R & =\frac{G_{4}}{2}\left[\left(\partial_{\mu} X \partial_{\nu} X-\frac{g_{\mu \nu}}{2} \partial_{\eta} X \partial^{\eta} X\right)\right. \\
& \left.+\left(\partial_{\mu} Y \partial_{\nu} Y-\frac{g_{\mu \nu}}{2} \partial_{\eta} Y \partial^{\eta} Y\right)-g_{\mu \nu} \tilde{V}(X, Y)\right],
\end{aligned}
$$

$\square X=\partial_{X} \tilde{V}$, and $\square Y=\partial_{Y} \tilde{V}$. In order to allow for a dS era we assume that the metric takes the form

$$
d s^{2}=-d t^{2}+e^{2 h(t)} d \vec{x}^{2}
$$

and that $X$ and $Y$ depend only on the time coordinate, i.e., $X=X(t)$ and $Y=Y(t)$. Then the equations of motion for $X$ and $Y$ can be written as

$$
\ddot{X}+3 \dot{h} \dot{X}=-\partial_{X} \tilde{V}
$$

and

$$
\ddot{Y}+3 \dot{h} \dot{Y}=-\partial_{Y} \tilde{V}
$$

whereas the only two independent components of Eq. (16) are

$$
\dot{h}^{2}=\frac{G_{4}}{6}\left[\frac{1}{2}\left(\dot{X}^{2}+\dot{Y}^{2}\right)+\tilde{V}(X, Y)\right]
$$

and

$$
2 \ddot{h}+3 \dot{h}^{2}=\frac{G_{4}}{2}\left[-\frac{1}{2}\left(\dot{X}^{2}+\dot{Y}^{2}\right)+\tilde{V}(X, Y)\right] .
$$

The terms in the square brackets in Eq. (15) take the form of a quadratic function of $e^{-\sqrt{G_{4}} X}$. This function has a global minimum at $e^{-\sqrt{G_{4}} X_{0}}=R_{2} r_{c}^{4} /\left(2 G_{6} b^{2}\right)$. Indeed, the necessary and sufficient condition for a minimum is that $R_{2}>0$, so hereafter we only consider the spherical compactification, where $e^{-\sqrt{G_{4}} X_{0}}=M_{\mathrm{Pl}}^{2} /\left(4 \pi b^{2}\right)$. The condition for the potential to show a dS rather than an AdS or Minkowski phase is $\xi b^{2}>1$. Now, we expand Eq. (15) around the minimum,

$$
\tilde{V}(X, Y)=\frac{e^{\sqrt{G_{4}} Y}}{G_{4}}\left[\mathcal{K}+\frac{{\overline{M_{X}}}^{2}}{2}\left(X-X_{0}\right)^{2}+\mathcal{O}\left(\left(X-X_{0}\right)^{3}\right)\right],
$$

where

$$
\overline{M_{X}} \equiv \frac{1}{\sqrt{\pi} b r_{c}}
$$


and

$$
\mathcal{K} \equiv \frac{M_{\mathrm{Pl}}^{2}}{4 \pi r_{c}^{2} b^{2}}\left(b^{2} \xi-1\right)
$$

As shown by Salam-Sezgin [9] the requirements for preserving a fraction of supersymmetry (SUSY) in spherical compactifications to four dimension imply $b^{2} \xi=1$, corresponding to winding number $n= \pm 1$ for the monopole configuration. Consequently, a ( $Y$-dependent) dS background can be obtained only through SUSY breaking. For now we will leave open the symmetry breaking mechanism and come back to this point after our phenomenological discussion. The $Y$-dependent physical mass of the $X$-particles at any time is

$$
M_{X}(Y)=\frac{e^{\sqrt{G_{4}} Y / 2}}{\sqrt{G_{4}}} \overline{M_{X}},
$$

which makes this a varying mass particle (VAMP) model [16], although, in this case, the dependence on the quintessence field is fixed by the theory. The dS (vacuum) potential energy density is

$$
V_{Y}=\frac{e^{\sqrt{G_{4}} Y}}{G_{4}} \mathcal{K} .
$$

In general, classical oscillations for the $X$ particle will occur for

$$
M_{X}>H=\sqrt{\frac{G_{4} \rho_{\mathrm{tot}}}{3}},
$$

where $\rho_{\text {tot }}$ is the total energy density. (This condition is well known from axion cosmology [19]). A necessary condition for this to hold can be obtained by saturating $\rho$ with $V_{Y}$ from Eq. (26) and making use of Eqs. (23) to (27), which leads to $\xi b^{2}<7$. Of course, as we stray from the present into an era where the $\mathrm{dS}$ energy is not dominant, we must check at every step whether the inequality (27) holds. If the inequality is violated, the $X$-particle ceases to behave like CDM.

In what follows, some combination of the parameters of the model will be determined by fitting present cosmological data. To this end we assume that SM fields are confined to $N_{1}$ and we denote with $\rho_{\text {rad }}$ the radiation energy, with $\rho_{X}$ the matter energy associated with the $X$-particles, and with $\rho_{\text {mat }}$ the remaining matter density. With this in mind, Eq. (19) can be re-written as

$$
\ddot{Y}+3 H \dot{Y}=-\frac{\partial V_{\text {eff }}}{\partial Y}
$$

where $V_{\text {eff }} \equiv V_{Y}+\rho_{X}$ and $H$ is defined by the Friedmann equation

$$
H^{2} \equiv \dot{h}^{2}=\frac{1}{3 M_{\mathrm{Pl}}^{2}}\left[\frac{1}{2} \dot{Y}^{2}+V_{\mathrm{eff}}+\rho_{\mathrm{rad}}+\rho_{\mathrm{mat}}\right] .
$$

(Note that the matter energy associated to the $X$ particles is contained in $V_{\text {eff. }}$ )

It is more convenient to consider the evolution in $u \equiv-\ln (1+z)$, where $z$ is the redshift parameter. As long as the oscillation condition is fulfilled, the VAMP CDM energy density is given in terms of the $X$-particle number density $n_{X}[20]$

$$
\rho_{X}(Y, u)=M_{X}(Y) n_{X}(u)=C e^{\sqrt{G_{4}} Y / 2} e^{-3 u},
$$


where $C$ is a constant to be determined by fitting to data. Along with Eq. (26), these define for us the effective ( $u$-dependent) VAMP potential

$$
V_{\text {eff }}(Y, u) \equiv V_{Y}+\rho_{X}=A e^{\sqrt{G_{4}} Y}+C e^{\sqrt{G_{4}} Y / 2} e^{-3 u},
$$

where a $A$ is just a constant given in terms of model parameters through Eqs. (22) and (24).

Hereafter we adopt natural units, $M_{\mathrm{Pl}}=1$. Denoting by a prime derivatives with respect to $u$, the equation of motion for $Y$ becomes

$$
\frac{Y^{\prime \prime}}{1-Y^{\prime 2} / 6}+3 Y^{\prime}+\frac{\partial_{u} \rho Y^{\prime} / 2+3 \partial_{Y} V_{\mathrm{eff}}}{\rho}=0,
$$

where $\rho=V_{\text {eff }}+\rho_{\text {rad }}+\rho_{\text {mat }}$. Quantities of importance are the dark energy density

$$
\rho_{Y}=\frac{1}{2} H^{2} Y^{\prime 2}+V_{Y}
$$

generally expressed in units of the critical density $\left(\Omega \equiv \rho / \rho_{\mathrm{c}}\right)$

$$
\Omega_{Y}=\frac{\rho_{Y}}{3 H^{2}}
$$

and the Hubble parameter

$$
H^{2}=\frac{\rho}{3-Y^{\prime 2} / 2}
$$

The equation of state is

$$
w_{Y}=\left[\frac{H^{2} Y^{\prime 2}}{2}-V_{Y}\right]\left[\frac{H^{2} Y^{\prime 2}}{2}+V_{Y}\right]^{-1} .
$$

We pause to note that the exponential potential $V_{Y} \sim e^{\lambda Y / M_{\mathrm{Pl}}}$, with $\lambda=\sqrt{2}$. Asymptotically, this represents the crossover situation with $w_{Y}=-1 / 3$ [22], implying expansion at constant velocity. Nevertheless, we will find that there is a brief period encompassing the recent past $(z \lesssim 6)$ where there has been significant acceleration.

Returning now to the quantitative analysis, we take $\rho_{\text {mat }}=B e^{-3 u}$ and $\rho_{\text {rad }}=$ $10^{-4} \rho_{\text {mat }} e^{-u} f(u)$ [21] where $B$ is a constant and $f(u)$ parameterizes the $u$-dependent number of radiation degrees of freedom. In order to interpolate the various thresholds appearing prior to recombination (among others, QCD and electroweak), we adopt a convenient phenomenological form $f(u)=\exp (-u / 15)$ [23]. We note at this point that solutions of Eq. (32) are independent by an overall normalization for the energy density. This is also true for the dimensionless quantities of interest $\Omega_{Y}$ and $w_{Y}$.

With these forms for the energy densities, Eq. (32) can be integrated for various choices of $A, B$, and $C$, and initial conditions at $u=-30$. We take as initial condition $Y(-30)=0$. Because of the slow variation of $Y$ over the range of $u$, changes in $Y(-30)$ are equivalent to altering the quantities $A$ and $C$ [24]. In accordance to equipartition arguments [24, 25] we take $Y^{\prime}(-30)=0.08$. Because the $Y$ evolution equation depends only on energy density ratios, and hence only on the ratios $A: B: C$ of the previously introduced constants, we may, for the purposes of integration and without loss of generality, arbitrarily fix $B$ and then scan the $A$ and $C$ parameter space for applicable solutions. In Fig. 1 we show a sample qualitative fit to the data. It has the property of allowing the maximum value of $X$-CDM 

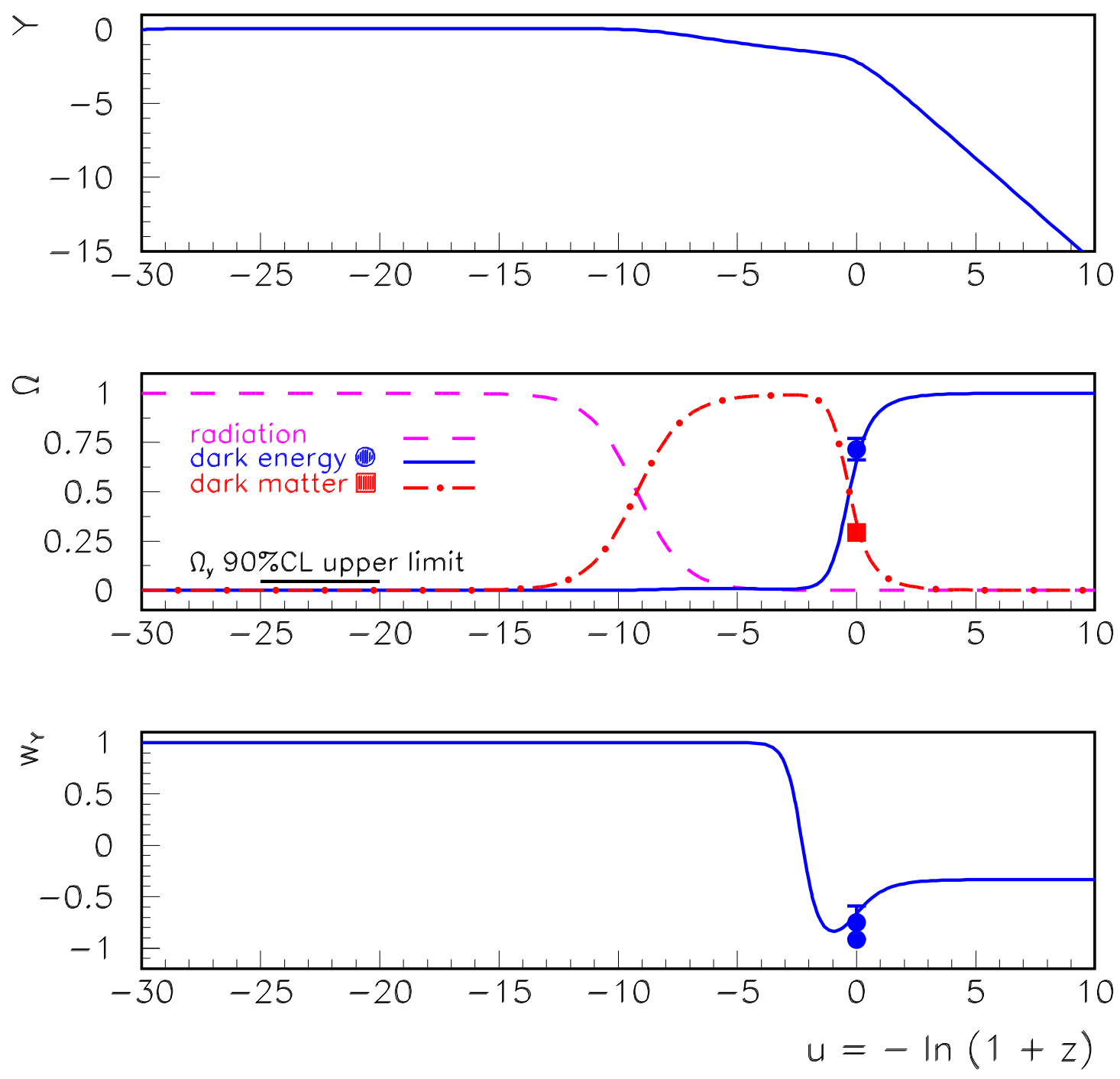

FIG. 1: The upper panel shows the evolution of $Y$ as a function of $u$. Today corresponds to $z=0$ and for primordial nucleosynthesis $z \approx 10^{10}$. We set the initial conditions $Y(-30)=0$ and $Y^{\prime}(-30)=0.08$; we take $A: B: C=11: 0.3: 0.1$. The second panel shows the evolution of $\Omega_{Y}$ (solid line), $\Omega_{\text {mat }}$ (dot-dashed line), and $\Omega_{\text {rad }}$ (dashed line) superposed over experimental best fits from SDSS and WMAP observations $[13,14]$. The curves are not actual fits to the experimental data but are based on the particular choice of the $Y$ evolution shown in the upper panel, which provides eyeball agreement with existing astrophysical observations. The lower panel shows the evolution of the equation of state $w_{Y}$ superposed over the best fits to WMAP + SDSS data sets and WMAP + SNGold [14] . The solution of the field equations is consistent with the requirement from primordial nucleosynthesis, $\Omega_{Y}<0.045$ (90\%CL) [12], it also shows the established radiation and matter dominated epochs, and at the end shows an accelerated dS era.

(about $7 \%$ of the total dark matter component) before the fits deviate unacceptably from data.

It is worth pausing at this juncture to examine the consequences of this model for variation in the fine structure constant and long range forces. Specifically, excitations of the 
electromagnetic field on $N_{1}$ will, through the presence of the dilaton factor in Eq. (2), seemingly induce variation in the electromagnetic fine structure constant $\alpha_{\mathrm{em}}=e^{2} / 4 \pi$, as well as a violation of the equivalence principle through a long range coupling of the dilaton to the electromagnetic component of the stress tensor. We now show that these effects are extremely negligible in the present model. First, it is easily seen using Eqs. (2) and (3) together with Eqs. (8)-(15), that the electromagnetic piece of the lagrangian as viewed from $N_{1}$ is

$$
\mathcal{L}_{\mathrm{em}}=-\frac{2 \pi}{4} e^{-\sqrt{G_{4}} X} \widetilde{f}_{\mu \nu}^{2},
$$

where $\widetilde{f}_{\mu \nu}$ denotes a quantum fluctuation of the electromagnetic $U(1)$ field. (Fluctuations of the $U(1)$ background field are studied in the Appendix). At the equilibrium value $X=X_{0}$, the exponential factor is

$$
e^{-\sqrt{G_{4}} X_{0}}=\frac{M_{\mathrm{Pl}}^{2}}{4 \pi b^{2}}
$$

so that we can identify the electromagnetic coupling $\left(1 / e^{2}\right) \simeq M_{\mathrm{Pl}}^{2} / b^{2}$. This shows that $b \sim M_{\mathrm{Pl}}$. We can then expand about the equilibrium point, and obtain an additional factor of $\left(X-X_{0}\right) / M_{\mathrm{Pl}}$. This will do two things [26]: (a) At the classical level, it will induce a variation of the electromagnetic coupling as $X$ varies, with $\Delta \alpha_{\mathrm{em}} / \alpha_{\mathrm{em}} \simeq\left(X-X_{0}\right) / M_{\mathrm{Pl}}$; (b) at the quantum level, exchange of $X$ quanta will induce a new force through coupling to the electromagnetic component of matter.

Item $(b)$ is dangerous if the mass of the exchanged quanta are small, so that the force is long range. This is not the case in the present model: from Eq. (22) the $X$ quanta have mass of $\mathcal{O}\left(\bar{M}_{X} M_{\mathrm{Pl}}\right) \sim M_{\mathrm{Pl}} /\left(r_{c} b\right)$, so that if $r_{c}$ is much less than $\mathcal{O}(\mathrm{cm})$, the forces will play no role in the laboratory or cosmologically.

As far as the variation of $\alpha_{\mathrm{em}}$ is concerned, we find that $\rho_{X} / \rho_{\text {mat }}=(C / B) e^{Y / \sqrt{2}}$, so that

$$
\begin{aligned}
\rho_{X} & \simeq 3 \times 10^{-120} e^{-3 u} M_{\mathrm{Pl}}^{4} e^{Y / \sqrt{2}} \\
& =\frac{1}{4} \bar{M}_{X}^{2}\left(X-X_{0}\right)^{2} e^{Y \sqrt{2}} M_{\mathrm{Pl}}^{2} .
\end{aligned}
$$

This then gives,

$$
\sqrt{\left\langle\left(X-X_{0}\right)^{2}\right\rangle} \equiv \Delta X_{\mathrm{rms}} \approx 10^{-60} e^{-3 u / 2} M_{\mathrm{Pl}} e^{Y /(2 \sqrt{2})} / \bar{M}_{X}
$$

During the radiation era, $Y \simeq$ const $\simeq 0$ (see Fig. 1), so that during nucleosynthesis $(u \simeq-23) \Delta X_{\mathrm{rms}} / M_{\mathrm{Pl}} \simeq 10^{-45} / \bar{M}_{X}$, certainly no threat. It is interesting that such a small value can be understood as a result of inflation: from the equation of motion for the $X$ field, it is simple to see that during a dS era with Hubble constant $H$, the amplitude $\Delta X_{\text {rms }}$ is damped as $e^{-3 H t / 2}$. For $50 e$-foldings, this represents a damping of $10^{32}$. In order to make the numbers match (assuming a pre-inflation value $\Delta X_{\mathrm{rms}} / M_{\mathrm{Pl}} \sim 1$ ) an additional damping of $\sim 10^{13}$ is required from reheat temperature to primordial nucleosynthesis. With the $e^{-3 u / 2}$ behavior, this implies a low reheat temperature, about $10^{6} \mathrm{GeV}$. Otherwise, one may just assume an additional fine-tuning of the initial condition on $X$.

As mentioned previously, the solutions of Eq. (32), as well as the quantities we are fitting to $\left(\Omega_{Y}\right.$ and $\left.w_{Y}\right)$, depend only on the ratios of the energy densities. From the eyeball fit in Fig. 1 we have, up to a common constant, $\rho_{\text {ordinary matter }} \equiv \rho_{\text {mat }} \propto 0.3 e^{-3 u}$ and $V_{Y} \propto 11 e^{\sqrt{2} Y}$. We can deduce from these relations that

$$
\frac{V_{Y}(\text { now })}{\rho_{\text {mat }}(\text { now })}=\frac{11}{0.3} e^{\sqrt{2} Y(\text { now })} \simeq 36 e^{\sqrt{2} Y(\text { now })} .
$$


Besides, we know that $\rho_{\text {mat }}($ now $) \simeq 0.3 \rho_{c}$ (now) $\simeq 10^{-120} M_{\mathrm{Pl}}^{4}$. Now, Eqs. (22) and (24) lead to

$$
V_{Y}(\text { now })=e^{\sqrt{2} Y(\text { now })} \frac{M_{\mathrm{Pl}}^{4}}{8 \pi r_{c}^{2} b^{2}}\left(b^{2} \xi-1\right)
$$

so that from Eqs. (41) and (42) we obtain

$$
\frac{1}{8 \pi r_{c}^{2} b^{2}}\left(b^{2} \xi-1\right) \simeq 10^{-119}
$$

It is apparent that this condition cannot be naturally accomplished by choosing large values of $r_{c}$ and/or $b$. There remains the possibility that SUSY breaking [27] or non-perturbative effects lead to an exponentially small deviation of $b^{2} \xi$ from unity, such that $b^{2} \xi=1+$ $\mathcal{O}\left(10^{-119}\right)[29]$. Since a deviation of $b^{2} \xi$ from unity involves a breaking of supersymmetry, a small value for this dimensionless parameter, perhaps $\left(1 \mathrm{TeV} / M_{\mathrm{Pl}}\right)^{2} \sim 10^{-31}$, can be expected on the basis of 't Hooft naturalness. It is the extent of the smallness, of course, which remains to be explained.

\section{THE STRING CONNECTION}

We now briefly comment on how the six dimensional solution derived above reads in String theory. To this end, we use the uplifting formulae developed by Cvetic, Gibbons and Pope [10]; we will denote with the subscript "cgp" the quantities of that paper and with "us" quantities in our paper. Let us more specifically look at Eq. (34) in Ref. [10], where the authors described the six dimensional Lagrangian they uplifted to Type I String theory. By simple inspection, we can see that the relation between their variables and fields with the ones we used in Eq. (2) is $\left.\phi\right|_{\text {cgp }}=-\left.2 \phi\right|_{\text {us }},\left.F_{2}\right|_{\text {cgp }}=\left.\sqrt{G_{6}} F_{2}\right|_{\text {us }},\left.H_{3}\right|_{\text {cgp }}=\left.\sqrt{G_{6} / 3} G_{3}\right|_{\text {us }}$, and $\left.\bar{g}^{2}\right|_{\text {cgp }}=\xi /\left.\left(8 G_{6}\right)\right|_{\text {us }}$. Our six dimensional background is determined by the (string frame) metric $d s_{6}^{2}=e^{2 f}\left[-d t^{2}+e^{2 h} d x_{3}^{2}+r_{c}^{2} d{\sigma_{2}}^{2}\right]$, the gauge field $F_{\vartheta \varphi}=-b \sin \vartheta$, and the $t$ dependent functions $h(t), f(t)=\sqrt{G_{4}}(X-Y) / 4$, and $\phi(t)=\sqrt{G_{4}}(X+Y) / 2$. Identifying these expressions with those in Eqs. (47), (48) and (49) of Ref. [10] one obtains a full Type I or Type IIB configuration, consisting of a 3 -form (denoted by $F_{3}$ ),

$$
\begin{aligned}
F_{3} & =\frac{8 G_{6} \sinh \hat{\rho} \cosh \hat{\rho}}{\xi \cosh 2 \hat{\rho}} d \hat{\rho} \wedge\left(d \alpha-\sqrt{\frac{\xi}{8 G_{6}}} b \cos \vartheta d \varphi\right) \wedge\left(d \beta+\sqrt{\frac{\xi}{8 G_{6}}} b \cos \vartheta d \varphi\right) \\
& -\frac{\sqrt{2} G_{6} b}{\sqrt{\xi} \cosh 2 \hat{\rho}} \sin \vartheta d \theta \wedge d \varphi \wedge\left[\cosh ^{2} \hat{\rho}\left(d \alpha-\sqrt{\frac{\xi}{8 G_{6}}} b \cos \vartheta d \varphi\right)\right. \\
& \left.-\sinh ^{2} \hat{\rho}\left(d \beta+\sqrt{\frac{\xi}{8 G_{6}}} b \cos \vartheta d \varphi\right)\right],
\end{aligned}
$$

a dilaton (denoted by $\hat{\phi})$

$$
e^{2 \hat{\phi}}=\frac{e^{2 \phi}}{\cosh (2 \hat{\rho})}
$$

and a ten dimensional metric that in the string frame reads

$$
d s_{\mathrm{str}}^{2}=e^{\phi} d s_{6}^{2}+d z^{2}+\frac{4 G_{6}}{\xi}\left[d \hat{\rho}^{2}+\frac{\cosh ^{2} \hat{\rho}}{\cosh 2 \hat{\rho}}\left(d \alpha-\sqrt{\frac{\xi}{8 G_{6}}} b \cos \vartheta d \varphi\right)^{2}\right.
$$




$$
\left.+\frac{\sinh ^{2} \hat{\rho}}{\cosh 2 \hat{\rho}}\left(d \beta+\sqrt{\frac{\xi}{8 G_{6}}} b \cos \vartheta d \varphi\right)^{2}\right],
$$

where $\hat{\rho}, z, \alpha$, and $\beta$ denote the four extra coordinates. It is important to stress that though the uplifted procedure decribed above implies a non-compact internal manifold, the metric in Eq. (46) can be interpreted within the context of [7] (i.e., $0 \leq \hat{\rho} \leq L$, with $L \gg 1$ an infrared cutoff where the spacetime smoothly closes up) to obtain a finite volume for the internal space and consequently a non-zero but tiny value for $G_{6}$.

\section{CONCLUSIONS}

We studied the six dimensional Salam-Sezgin model [9], where a solution of the form Minkowski ${ }_{4} \times S^{2}$ is known to exist, with a $U(1)$ monopole serving as background in the twosphere. This model circumvents the hypotheses of the no-go theorem [4] and then when lifted to String theory can show a dS phase. In this work we have allowed for time dependence of the six-dimensional moduli fields and metric (with a Robertson-Walker form). Time dependence in these fields vitiates invariance under the supersymmetry transformations. With these constructs, we have obtained the following results:

(1) In terms of linear combinations of the $S^{2}$ moduli field and the six dimensional dilaton, the effective potential consists of $(a)$ a pure exponential function of a quintessence field (this piece vanishes in the supersymmetric limit of the static theory) and (b) a part which is a source of cold dark matter, with a mass proportional to an exponential function of the quintessence field. This presence of a VAMP CDM candidate is inherent in the model.

(2) If the monopole strength is precisely at the value prescribed by supersymmetry, the model is in gross disagreement with present cosmological data - there is no accelerative phase, and the contribution of energy from the quintessence field is purely kinetic. However, a miniscule deviation of $\mathcal{O}\left(10^{-120}\right)$ from this value permits a qualitative match with data. Contribution from the VAMP component to the matter energy density can be as large as about $7 \%$ without having negative impact on the fit. The emergence of a VAMP CDM candidate as a necessary companion of dark energy has been a surprising aspect of the present findings, and perhaps encouraging for future exploration of candidates which can assume a more prominent role in the CDM sector.

(3) In our model, the exponential potential $V_{Y} \sim e^{\lambda Y / M_{\mathrm{Pl}}}$, with $Y$ the quintessence field and $\lambda=\sqrt{2}$. The asymptotic behavior of the scale factor for exponential potentials $e^{h(t)} \approx t^{2 / \lambda^{2}}$, so that for our case $h \approx \ln t$, leading to a conformally flat Robertson-Walker metric for large times. The deviation from constant velocity expansion into a brief accelerated phase in the neighborhood of our era makes the model phenomenologically viable. In the case that the supersymmetry condition $\left(b^{2} \xi=1\right)$ is imposed, and there is neither radiant energy nor dark matter except for the $X$ contribution, we find for large times that the scale parameter $e^{h(t)} \approx \sqrt{t}$, so that even in this case the asymptotic metric is Robertson-Walker rather than Minkowski. Moreover, and rather intriguingly, the scale parameter is what one would find with radiation alone [28].

In sum, in spite of the shortcomings of the model (not a perfect fit, requirement of a tiny deviation from supersymmetric prescription for the monopole embedding), it has provided a stimulating new, and unifying, look at the dark energy and dark matter puzzles. 


\section{Acknowledgments}

We would like to thank Costas Bachas and Roberto Emparan for valuable discussions. The research of HG was supported in part by the National Science Foundation under Grant No. PHY-0244507.

\section{APPENDIX}

In this appendix we study the quantum fluctuations of the $U(1)$ field associted to the background configuration. We start by considering fluctuations of the background field $A_{M}^{0}$ in the 4 dimensional space, i.e,

$$
A_{M} \rightarrow A_{M}^{0}+\epsilon a_{M},
$$

where $A_{M}^{0}=0$ if $M \neq \varphi$ and $a_{M}=0$ if $M=\vartheta, \varphi$. The fluctuations on $A_{M}^{0}$ lead to

$$
F_{M N} \rightarrow F_{M N}^{0}+\epsilon f_{M N}
$$

Then,

$$
F_{M N} F^{M N}=g^{M L} g^{N P}\left[F_{M N}^{0} F_{L P}^{0}+\epsilon F_{M N}^{0} f_{L P}+\epsilon^{2} f_{M N} f_{L P}\right] .
$$

The second term vanishes and the first and third terms are nonzero because $F_{M N}^{0} \neq 0$ in the compact space and $f_{M N} \neq 0$ in the 4 dimensional space. If the Kalb-Ramond potential $B_{N M}=0$, then the 3 -form field strength can be written as

$$
G_{M N P}=\kappa A_{[M} F_{N P]}=\frac{\kappa}{3 !}\left[A_{M} F_{N P}+A_{P} F_{M N}-A_{N} F_{M P}\right] .
$$

Now we introduce notation of differential forms, in which the usual Maxwell field and field strenght read

$$
A_{1}=A_{M} d x^{M} \text { and } F_{2}=F_{M N} d x^{M} \wedge d x^{N} ;
$$

respectively. (Note that $d x^{M} \wedge d x^{N}$ is antisymmetrized by definition.) With this in mind the 3 -form reads

$$
G_{3}=\kappa A_{1} \wedge F_{2}=\kappa A_{M} F_{N P} d x^{M} \wedge d x^{N} \wedge d x^{P} .
$$

Substituting Eqs. (47) and (48) into Eq. (52) we obtain

$$
G_{3}=\kappa\left[\left(A_{M}^{0}+\epsilon a_{M}\right)\left(F_{N P}^{0}+\epsilon f_{N P}\right) d x^{M} \wedge d x^{N} \wedge d x^{P}\right] .
$$

The background fields read

$$
A_{1}^{0}=b \cos \vartheta d \varphi, \quad F_{2}^{0}=-b \sin \vartheta d \vartheta \wedge d \varphi,
$$

and the fluctuations on the probe brane become

$$
a_{1}=a_{\mu} d x^{\mu}, \quad f_{2}=f d x^{\mu} \wedge d x^{\nu}, \text { with } f=\partial_{\mu} a_{\nu}-\partial_{\mu} a_{\nu} .
$$

All in all,

$$
\begin{aligned}
\frac{G_{3}}{\kappa} & =A_{\varphi}^{0} F_{\vartheta \varphi}^{0} d \varphi \wedge d \vartheta \wedge d \varphi+\epsilon A_{\varphi}^{0} f_{\mu \nu} d \varphi \wedge d x^{\mu} \wedge d x^{\nu}+\epsilon F_{\vartheta \varphi}^{0} a_{\mu} d \vartheta \wedge d \varphi \wedge d x^{\mu} \\
& +\epsilon^{2} a_{\mu} f_{\zeta \nu} d x^{\mu} \wedge d x^{\zeta} \wedge d x^{\nu}
\end{aligned}
$$


Using Eq. (54) and the antisymmetry of the wedge product, Eq. (56) can be re-written as

$$
\frac{G_{3}}{\kappa}=\epsilon\left[b \cos \vartheta f_{\mu \nu} d \varphi \wedge d x^{\mu} \wedge d x^{\nu}-b a_{\mu} \sin \vartheta d \vartheta \wedge d \varphi \wedge d x^{\mu}+\epsilon a_{\mu} f_{\zeta \nu} d x^{\mu} \wedge d x^{\zeta} \wedge d x^{\nu}\right] .
$$

From the metric

$$
d s^{2}=e^{2 \alpha} d x_{4}^{2}+e^{2 \beta}\left(d \vartheta^{2}+\sin \vartheta^{2} d \varphi^{2}\right)
$$

we can write the vielbeins

$$
\begin{aligned}
& e^{a}=e^{\alpha} d x^{a}, \quad e^{\vartheta}=e^{\beta} d \vartheta, \quad e^{\varphi}=e^{\beta} \sin \vartheta d \varphi, \\
& d x^{a}=e^{-\alpha} e^{a}, \quad d \vartheta=e^{-\beta} e^{\vartheta}, \quad d \varphi=\frac{e^{-\beta}}{\sin \vartheta} e^{\varphi}
\end{aligned}
$$

where $\beta \equiv f+\ln r_{c}$. (Lower latin indeces from the beginning of the alphabet indicate coordinates associted to the four dimensional Minkowski spacetime with metric $\eta_{a b}$.) Substituting into Eq. (57) we obtain

$$
\frac{G_{3}}{\kappa}=\epsilon\left[b \frac{\cos \vartheta}{\sin \vartheta} e^{-2 \alpha-\beta} f_{a b} e^{\varphi} \wedge e^{a} \wedge e^{b}-b e^{-\alpha-2 \beta} a_{a} e^{\vartheta} \wedge e^{\varphi} \wedge e^{a}+\epsilon e^{-3 \alpha} a_{a} f_{c b} e^{a} \wedge e^{c} \wedge e^{b}\right]
$$

where $f_{a b}=\partial_{a} a_{b}-\partial_{b} a_{a}$. Because the three terms are orthogonal to each other straightforward calculation leads to

$$
G_{3}^{2}=\kappa^{2} \epsilon^{2}\left(b^{2} \cot ^{2} \vartheta e^{-4 \alpha-2 \beta} f_{a b}^{2}+b^{2} e^{-2 \alpha-4 \beta} a_{a}^{2}\right)+\mathcal{O}\left(\epsilon^{4}\right) .
$$

Then, the 5th term in Eq. (2) can be written as

$$
\begin{aligned}
S_{G_{3}} & =-\frac{1}{2 G_{6}} \int d^{4} x \frac{G_{6}}{6} e^{4 \alpha+2 \beta} \sqrt{\eta_{4}} e^{-2 \phi} \int d \vartheta d \varphi \sin \vartheta\left[\left(\kappa^{2} \epsilon^{2} b^{2} \cot ^{2} \vartheta e^{-4 \alpha-2 \beta}\right) f_{a b}^{2}\right. \\
& \left.+\left(\kappa^{2} \epsilon^{2} b^{2} e^{-2 \alpha-4 \beta}\right) a_{a}^{2}\right]
\end{aligned}
$$

whereas the contribution from the 4 th term in Eq. (2) can be computed from Eq. (49) yielding

$$
\begin{aligned}
S_{F_{2}} & =-\frac{1}{2 G_{6}} \int d^{4} x \sqrt{\eta_{4}} 2 \pi e^{2 \beta-\phi} G_{6} \epsilon^{2} f_{a b}^{2} \\
& =-\int d^{4} x \sqrt{\eta_{4}} \pi e^{2 f-\phi} r_{c}^{2} \epsilon^{2} f_{a b}^{2} .
\end{aligned}
$$

Thus,

$$
S_{G_{3}}+S_{F_{2}}=-\int d^{4} x\left[\frac{1}{4 g^{2}} f_{a b}^{2}+\frac{m^{2}}{2} a_{a}^{2}\right],
$$

where the four dimensional effective coupling and the effective mass are of the form

$$
\frac{1}{g^{2}}=4 \epsilon^{2} \sqrt{\eta_{4}}\left[\pi e^{2 f-\phi} r_{c}^{2}+\frac{1}{12} \kappa^{2} b^{2} e^{-2 \phi} \int d \vartheta d \varphi \sin \vartheta \cot ^{2} \vartheta\right] \rightarrow \infty
$$

and

$$
m^{2}=\frac{2}{3} \pi \kappa^{2} b^{2} \epsilon^{2} e^{2 \alpha-2 \beta-2 \phi}
$$


For the moment we let $\int d \vartheta d \varphi \sin \vartheta \cot ^{2} \vartheta=N$, where eventually we set $N \rightarrow \infty$. Now to make quantum particle identification and coupling, we carry out the transformation $a_{a} \rightarrow g \hat{a}_{a}$ [30]. This implies that the second term in the right hand side of Eq. (64) vanishes, yielding

$$
f_{a b}=\partial_{a}\left(g \hat{a}_{b}\right)-\partial_{b}\left(g \hat{a}_{a}\right)=\partial_{a} g \hat{a}_{b}-\partial_{b} g \hat{a}_{a}+g \partial_{a} \hat{a}_{b}-g \partial_{b} \hat{a}_{a}=g \hat{f}_{a b}+\hat{a} \wedge d g
$$

and consequently to leading order in $N$

$$
\frac{1}{g^{2}} f_{a b}^{2}=\frac{1}{g^{2}}\left[g^{2} \hat{f}_{a b}^{2}+(\hat{a} \wedge d g)^{2}+2 g \hat{a}_{b} \hat{f}^{a b} \partial_{a} g\right] .
$$

If the coupling depends only on the time variable,

$$
\frac{1}{g^{2}} f_{a b}^{2} \rightarrow \hat{f}_{a b}^{2}+\left(\frac{\dot{g}}{g}\right)^{2} \hat{a}_{a}^{2}+2 \frac{\dot{g}}{g} \hat{a}_{i} \hat{f}^{t i}
$$

where $\dot{g}=\partial_{t} g$ and lower latin indices from the middle of the alphabet refer to the brane space-like dimensions. If we choose a time-like gauge in which $a_{t}=0$, then the term $(\dot{g} / g) \hat{a}_{i} \hat{f}^{t i}$ can be written as $(1 / 2)(\dot{g} / g)(d / d t)\left(\hat{a}_{i}\right)^{2}$, which after an integration by parts gives $-(1 / 2)[(d / d t)(\dot{g} / g)] \hat{a}_{i}^{2}$; with $g \sim e^{-\phi}$, the factor in square brackets becomes $-\ddot{\phi}$. Since $\phi=\sqrt{G_{4}}(X+Y)$, the rapidly varying $\ddot{X}$ will average to zero, and one is left just with the very small $\ddot{Y}$, which is of order Hubble square. For the term $(\dot{g} / g)^{2}\left(a_{i}\right)^{2}$, the term $(\dot{X})^{2}$ also averages to order Hubble square, implying that the induced mass term is of horizon size. These "paraphotons" carry new relativistic degrees of freedom, which could in turn modify the Hubble expansion rate during Big Bang nucleosynthesis (BBN). Note, however, that these extremely light gauge bosons are thought to be created through inflaton decay and their interactions are only relevant at Planck-type energies. Since the quantum gravity era, all the paraphotons have been redshifting down without being subject to reheating, and consequently at BBN they only count for a fraction of an extra neutrino species in agreement with observations.

[1] A. G. Riess et al. [Supernova Search Team Collaboration], Astron. J. 116, 1009 (1998) [arXiv:astro-ph/9805201]; S. Perlmutter et al. [Supernova Cosmology Project Collaboration], Astrophys. J. 517, 565 (1999) [arXiv:astro-ph/9812133]; N. A. Bahcall, J. P. Ostriker, S. Perlmutter and P. J. Steinhardt, Science 284, 1481 (1999) [arXiv:astro-ph/9906463].

[2] S. Weinberg, Phys. Rev. Lett. 59, 2607 (1987).

[3] R. Bousso and J. Polchinski, JHEP 0006, 006 (2000) [arXiv:hep-th/0004134]; L. Susskind arXiv:hep-th/0302219; M. R. Douglas, JHEP 0305, 046 (2003) [arXiv:hep-th/0303194]; N. Arkani-Hamed and S. Dimopoulos, JHEP 0506, 073 (2005) [arXiv:hep-th/0405159]; M. R. Douglas and S. Kachru, arXiv:hep-th/0610102.

[4] J. M. Maldacena and C. Nunez, Int. J. Mod. Phys. A 16, 822 (2001) [arXiv:hep-th/0007018]; G. W. Gibbons, "Aspects of Supergravity Theories," lectures given at GIFT Seminar on Theoretical Physics, San Feliu de Guixols, Spain, 1984. Print-85-0061 (CAMBRIDGE), published in GIFT Seminar 1984:0123.

[5] G. W. Gibbons and C. M. Hull, arXiv:hep-th/0111072. 
[6] P. K. Townsend and M. N. R. Wohlfarth, Phys. Rev. Lett. 91, 061302 (2003) [arXiv:hepth/0303097]. See also, N. Ohta, Phys. Rev. Lett. 91, 061303 (2003) [arXiv:hep-th/0303238].

[7] S. B. Giddings, S. Kachru and J. Polchinski, Phys. Rev. D 66, 106006 (2002) [arXiv:hepth/0105097].

[8] S. Kachru, R. Kallosh, A. Linde and S. P. Trivedi, Phys. Rev. D 68, 046005 (2003) [arXiv:hepth/0301240].

[9] A. Salam and E. Sezgin, Phys. Lett. B 147, 47 (1984).

[10] M. Cvetic, G. W. Gibbons and C. N. Pope, Nucl. Phys. B 677, 164 (2004) [arXiv:hepth/0308026].

[11] See e.g., J. J. Halliwell, Nucl. Phys. B 286, 729 (1987); Y. Aghababaie, C. P. Burgess, S. L. Parameswaran and F. Quevedo, JHEP 0303, 032 (2003) [arXiv:hep-th/0212091]; Y. Aghababaie, C. P. Burgess, S. L. Parameswaran and F. Quevedo, Nucl. Phys. B 680, 389 (2004) [arXiv:hep-th/0304256]; G. W. Gibbons, R. Guven and C. N. Pope, Phys. Lett. B 595, 498 (2004) [arXiv:hep-th/0307238]; Y. Aghababaie et al., JHEP 0309, 037 (2003) [arXiv:hep-th/0308064].

[12] K. A. Olive, G. Steigman and T. P. Walker, Phys. Rept. 333, 389 (2000) [arXiv:astroph/9905320]; R. Bean, S. H. Hansen and A. Melchiorri, Nucl. Phys. Proc. Suppl. 110, 167 (2002) [arXiv:astro-ph/0201127].

[13] M. Tegmark et al. [SDSS Collaboration], Phys. Rev. D 69, 103501 (2004) [arXiv:astro$\mathrm{ph} / 0310723]$.

[14] D. N. Spergel et al. [WMAP Collaboration], arXiv:astro-ph/0603449.

[15] J. J. Halliwell, Phys. Lett. B 185, 341 (1987); B. Ratra and P. J. E. Peebles, Phys. Rev. D 37, 3406 (1988); P. G. Ferreira and M. Joyce, Phys. Rev. Lett. 79, 4740 (1997) [arXiv:astroph/9707286]; P. G. Ferreira and M. Joyce, Phys. Rev. D 58, 023503 (1998) [arXiv:astroph/9711102]; E. J. Copeland, A. R. Liddle and D. Wands, Phys. Rev. D 57, 4686 (1998) [arXiv:gr-qc/9711068].

[16] D. Comelli, M. Pietroni and A. Riotto, Phys. Lett. B 571, 115 (2003) [arXiv:hep-ph/0302080]; U. Franca and R. Rosenfeld, Phys. Rev. D 69, 063517 (2004) [arXiv:astro-ph/0308149].

[17] R. M. Wald, "General Relativity," (University of Chicago Press, Chicago, 1984).

[18] A similar expression was derived by J. Vinet and J. M. Cline, Phys. Rev. D 71, 064011 (2005) [arXiv:hep-th/0501098].

[19] J. Preskill, M. B. Wise and F. Wilczek, Phys. Lett. B 120, 127 (1983).

[20] M. B. Hoffman, arXiv:astro-ph/0307350.

[21] This assumption will be justified a posteriori when we find that $\rho_{X} \ll \rho_{\text {mat }}$.

[22] E. J. Copeland, A. R. Liddle and D. Wands, op. cit. in Ref. [15].

[23] L. Anchordoqui and H. Goldberg, Phys. Rev. D 68, 083513 (2003) [arXiv:hep-ph/0306084].

[24] U. J. Lopes Franca and R. Rosenfeld, JHEP 0210, 015 (2002) [arXiv:astro-ph/0206194].

[25] P. J. Steinhardt, L. M. Wang and I. Zlatev, Phys. Rev. D 59, 123504 (1999) [arXiv:astroph/9812313].

[26] S. M. Carroll, Phys. Rev. Lett. 81, 3067 (1998) [arXiv:astro-ph/9806099].

[27] Y. Aghababaie, C. P. Burgess, S. L. Parameswaran and F. Quevedo, op. cit. in Ref. [15].

[28] This comes from a behavior $Y \simeq-\sqrt{2} u$ (compatible with the equations of motion), when combined with the $e^{-3 u}$ in Eq. (30).

[29] Before proceeding, we remind the reader that the requirements for preserving a fraction of SUSY in spherical compactifications to four dimensions imply $b^{2} \xi=1$, corresponding to the winding number $n= \pm 1$ for the monopole configuration. In terms of the Bohm-Aharonov 
argument on phases, this is consistent with usual requirement of quantization of the monopole. The SUSY breaking has associated a non-quantized flux of the field supporting the two sphere. In other words, if we perform a Bohm-Aharonov-like interference experiment, some phase change will be detected by a $U(1)$ charged particle that circulates around the associated Dirac string. The quantization of fluxes implied the unobservability of such a phase, and so in our cosmological set up, the parallel transport of a fermion will be slightly path dependent. One possibility is that the non-compact $\rho$ coordinate (in the uplift to ten dimensions, see Sec. III) is the direction in which the Dirac string exists. Then the cutoff necessary on the physics at large $\rho$ will introduce a slight (time-dependent) perturbation on the flux quantization condition. We are engaged at present in exploring possibilities along this line.

[30] This is because the definition of the propagator with proper residue for correct Feyman rules in perturbation theory, and therefore also the couplings, needs to be consistent with the form of the Hamiltonian $=\sum_{k} \omega(k) a_{k}^{\dagger} a_{k}$, with $\left[a, a^{\dagger}\right]=1$. This in turn implies that the kinetic term in the Lagrangian has the canonical form, $(1 / 4) \hat{f}_{a b}^{2}$, with the usual expansion of the vector field $a_{a}$. 